\title{
Variations in Functional Outcome Stratified by Discharge Disposition and Oncological Treatment Groups After Acute Inpatient Rehabilitation for Brain Tumor Patients ${ }^{\S}$
}

\author{
Vishwa S. Raj ${ }^{*}$, Christine Groves, Hyung Kim, Chloe Bomberger and James H. Norton
}

Carolinas Rehabilitation, 1100 Blythe Boulevard, Charlotte, NC 28203, USA

\begin{abstract}
Objective: This study evaluated variations in functional outcome for patients participating in a multidisciplinary acute inpatient rehabilitation program specific for brain tumors at a free standing acute inpatient rehabilitation hospital.

Methods: Data was collected retrospectively for 45 patients with brain tumors admitted for inpatient rehabilitation from January 1, 2008 to May 1, 2009. Primary outcome measure included Functional Independence Measure (FIM) score on admission and discharge based on discharge disposition and acute oncological treatment group. Data was collected regarding length of stay and general patient demographic information.

Results: Patients receiving chemotherapy and radiation while participating in inpatient rehabilitation had the greatest statistically significant gains in $\triangle$ FIM, FIM-D, $\Delta$ FIM-T, discharge FIM-T, discharge FIM-M, and FIM-E. Patients who discharged to home for continued aggressive treatment had greatest gains in $\Delta$ FIM, FIM-D, $\Delta$ FIM-T, discharge FIM-T, $\triangle$ FIM-M, discharge FIM-M, and FIM-E. Correlation data was also established between several variables.

Conclusions: Inpatient rehabilitation stay for brain tumor patients resulted in improvements of FIM score for all disposition and acute oncological treatment groups, though statistically significant differences were noted within groups. Greatest improvements were noted for patients receiving both chemotherapy and radiation therapy and patients who discharged to home for continued aggressive oncological treatment.
\end{abstract}

Keywords: Acute inpatient rehabilitation, brain tumor, chemotherapy, discharge disposition, functional outcomes, radiation therapy.

\section{INTRODUCTION}

Brain tumors are a significant cause of both morbidity and mortality in the United States. In 2005, 43,800 new cases of primary central nervous system (CNS) brain tumors were diagnosed and malignant CNS tumors caused 13,000 deaths [1]. Standard forms of treatment for these types of tumors include radiation therapy, chemotherapy, and/or surgical resection when possible [2]. Recent advances in surgical resection for grade III and grade IV gliomas have led to an improved mean survival of 48.2 weeks, with grade III tumors specifically showing mean survival of 73.4 weeks. Variables that were associated with improved survival included younger age with concurrent treatment, full surgical resection compared to biopsy alone, and improved functional scores as measured by the Karnofsky Performance Scale [3]. Survival rates vary according to the pathology of the tumor and vary significantly [4]. Glioblastomas and astrocytomas are a majority of cancers of the brain, representing $53.6 \%$ and $26.6 \%$, whereas menigiomas represent $41.3 \%$ of other

*Address correspondence to this author at the Carolinas Rehabilitation, 1100 Blythe Boulevard, Charlotte, NC 28203 USA; Tel: 704355 9330; Fax: 704355 0709; E-mail: Vishwa.raj@carolinashealthcare.org

${ }^{\S}$ A portion of this data will be presented in poster format at the European Cancer Rehabilitation and Survivorship Symposium being held in Copenahgen, Denmark in 2012. central nervous system cancers. For menigiomas, astrocytomas, and glioblastomas respectively, survival rates are $87.7 \%, 62.3 \%$, and $31.7 \%$ (1 year) versus $69.5 \%, 35.8 \%$, $2.9 \%$ (5 year) [5]. However with new and promising surgical and medical techniques, individuals can expect improved survival rates and neurological recovery [6-8].

The rationale of rehabilitation programs for brain cancer patients addresses both physical and psychological approaches to treat and prevent the onset of symptoms associated with this diagnosis [9]. Functional deficits vary according to the location and mass effect of a tumor. Symptoms may be related to direct invasion or compression of the brain, as well as increased intracranial pressure, postoperative changes, radiation effects, and chemotherapy [10]. Several studies have shown that rehabilitation, in both the inpatient and outpatient settings, can improve functionality and quality of life (QOL) in the brain tumor patient [11]. Given the multitude of symptoms associated with brain tumors (such as cognitive impairment, paresis, sensory loss, neurogenic bowel and bladder, and dysphagia), much attention was devoted to comparative studies evaluating functional outcomes with other rehabilitation specific CNS diagnoses [12]. When controlling for patient specific variables, brain tumor patients were found to have positive changes in Functional Independence Measure (FIM) score, though the changes were not as substantial as that for individuals with traumatic brain injury (TBI) [13]. It has also 
been noted that patients with brain tumors generally have decreased length of stay (LOS) compared to TBI patients [14].

Similar results were found when comparing individuals suffering from stroke to those with brain tumors. Though changes in FIM score were less dramatic, brain tumor patients generally discharged from inpatient rehabilitation earlier with less disability and fewer concurrent medical complications. Using comparable measures other than FIM, improvement in locomotor function was similar between both groups [15]. Improved rates of discharge to home may have been driven by good family support and concerns about patient life expectancy $[14,16]$. Aggressive physical therapy has shown significant improvements in balance and coordination for patients with tumors in the posterior fossa and cerebellopontine angle [17]. Furthermore, dysphagia protocols related to those used in stroke have been shown to improve overall swallow for brain tumor patients, with an increased number of individuals discharged from speech therapy with regular diets [18]. Of note, patients evaluated in several of these studies did not receive chemotherapy or radiation therapy during their stays in acute inpatient rehabilitation $[15,16,19]$. For patients who did receive concurrent radiation therapy alone during inpatient rehabilitation, functional improvements were noted. However, patients receiving radiation were a minority of the samples evaluated, representing less than $20 \%$ of all individuals in these studies $[13,20]$.

The purpose of this study is to evaluate how functional outcomes differ based on discharge disposition and acute oncological treatment groups after individuals participate in a multidisciplinary acute inpatient rehabilitation program specifically designed for the brain tumor population. It is hypothesized that patients who received acute oncological treatment while receiving rehabilitation interventions had positive functional outcomes at the time of discharge. In addition it is postulated that after participating in a brain tumor specific rehabilitation program, most individuals would achieve functional improvement that would justify their discharge to home for pursuance of further aggressive treatment. Demographic information will also be collected to understand trends as they relate to patients admitted for acute inpatient rehabilitation with a concurrent brain tumor diagnosis. To our knowledge, this is the first study to stratify outcomes based on discharge disposition and acute oncological treatment groups which include both radiation and chemotherapy. Furthermore correlation data will help to establish relationships between LOS, age, and FIM scores through the inpatient rehabilitation stay. These results may have implications for clinical practice regarding the appropriateness of admission of these patients to inpatient rehabilitation units, given increased focus on medical necessity requirements per Medicare. In addition to traditional measures of outcome, we have also described data in terms of average individual FIM score $(\Delta \mathrm{FIM})$, which may be a useful alternative metric for evaluation of function.

\section{MATERIALS AND METHODS}

This retrospective review consists of brain tumor patients who were admitted for inpatient rehabilitation from January 1, 2008 to May 1, 2009 at a free standing acute inpatient rehabilitation hospital, associated with a tertiary acute care hospital and large regional cancer center. This study was approved by our Institutional Review Board.

Patient information was collected through chart review using an electronic data base that records functional outcomes for any and all patients receiving inpatient rehabilitation program at Carolinas Rehabilitation. All patients had completed inpatient rehabilitation programs prior to the initiation of this investigation, and the investigators were blinded to collection of data. All protected health information was recoded to maintain patient confidentiality during data analysis. Patients were identified by tumor diagnosis, and were categorized as benign and minimally malignant (meningioma, World Health Organization (WHO) grade I brain tumor, and WHO grade II brain tumor), early stage malignant tumor (newly diagnosed WHO grade III and IV primary brain tumor), or late stage malignant tumor (recurrent WHO grade IV primary brain tumor or cerebral metastasis from another organ system). Primary outcome measures were recorded with the FIM score, specifically at admission and discharge. Results are reported as average individual FIM upon admission and discharge (FIM-A and FIM-D respectively), utilizing $\triangle$ FIM score from admission to discharge to provide interpretation consistent with measures at our home institution, reports as presented by several national benchmarking organizations, and data that has been used by our institution in regional recovery and auditing procedures. We have also presented data in formats consistent with previously reported results, such as FIM total (FIM-T), FIM motor (FIM-M), FIM cognitive (FIM-C), and FIM efficiency (FIM-E).

Data regarding patient demographics were collected to better understand the relationships between outcomes and patient characteristics. Areas of particular interest included inpatient LOS, age, ancillary treatment during the inpatient rehabilitation stay (categorized as no treatment, chemotherapy alone, radiation therapy alone, or both chemotherapy and radiation therapy), discharge treatment plan (categorized as hospice care at home or at hospice house, palliative care at skilled nursing facility (SNF), or discharge to home with continued aggressive treatment), and primary funding source (categorized as private insurance, Medicare, or North Carolina Medicaid). The only chemotherapeutic agent administered in this group of patients was oral temozolomide, which is consistent with oncological standards of care for the time period of this review. Palliative care consults at SNF is common in our region for patients who cannot discharge directly to home or hospice. Medicare guidelines do not allow for hospice provision while receiving inpatient SNF services and acute oncological services often cannot be provided while at SNF.

Descriptive statistics including means and standard deviations, or counts and percentages, were calculated. For data measured on the ordinal scale or measured on the interval scale but not normally distributed, the KruskalWallis test was used. For nominal data, the chi square or Fisher's exact test was employed. Spearman's correlations were used to test for linear relationships between the variables measured on the interval or ordinal scale. $\mathrm{SAS}{ }^{\circledR}$, version 9.2 was used for all analyses. A two-tailed $p$-value of less than 0.05 was considered statistically significant. 


\section{RESULTS}

The medical records of 45 individuals were reviewed over the specified time course. Racial distribution of patients was categorized as Caucasian (78\%), African American (18\%), Asian/Indian Subcontinent (2\%), and Hispanic (2\%). Most patients utilized Medicare benefits as funding for acute inpatient rehabilitation $(64.5 \%)$, with private insurance (31\%) and North Carolina Medicaid (4.5\%) representing alternative sources. There was a statistically significant correlation between funding source and associated age of the patient $(\mathrm{p}<0.001)$, indicating that our Medicare population was generally age appropriate and not necessarily reflective of younger patients receiving Medicare disability benefit.

Our sample was predominantly male (56\%); mean age was 65 with a LOS of 18 days. Patients had an overall positive trend for $\triangle F I M, \Delta$ FIM-T, $\triangle$ FIM-M, and $\triangle$ FIM-C; FIM-E for all patients was 1.30 (Table 1). During the acute inpatient rehabilitation stay, more than half of the patients received concurrent oncological treatment for their condition (Table 2), and $11 \%$ of patients required emergent transfer to the acute care medical setting for further intervention. Over ninety percent of patients treated during this time frame were able to discharge to home, with a majority continuing aggressive oncological therapy at home immediately after discharge from acute inpatient rehabilitation (Table 3). Eighty-four percent of patients had sufficient family support to allow for discharge to a home setting. More than half of the patients treated had a diagnosis of malignant tumor (late stage malignant: $51 \%$; early stage malignant: $24.5 \%$, minimally malignant and benign: $24.5 \%$ ), and $71 \%$ of all patients underwent gross surgical resection as part of their acute care treatment plan. Only $41 \%$ of patients were receiving concurrent treatment with antidepressants and $71 \%$ did not receive any forms of neurostimulation.

Our results confirmed statistical significance when comparing the role of acute oncological treatment while in rehabilitation to discharge disposition $(p<0.009$; Table 4).

Table 1. Overall Age, LOS, and FIM Averages for Individuals Participating in a Brain Tumor Specific Inpatient Rehabilitation Program

\begin{tabular}{|c|c|c|c|c|c|}
\hline & Mean & Std Deviation & Median & Minimum & Maximum \\
\hline Age & 65.18 & 14.90 & 70.00 & 32 & 88 \\
\hline FIM-A & 3.57 & 0.83 & 3.48 & 1.67 & 5.31 \\
\hline FIM-D & 4.56 & 0.87 & 4.74 & 2.27 & 5.88 \\
\hline Admission FIM-T & 61.13 & 14.40 & 60.00 & 29.00 & 94.00 \\
\hline Discharge FIM-T & 80.56 & 15.55 & 85.00 & 40.00 & 105.00 \\
\hline$\Delta$ FIM-T & 19.42 & 12.56 & 21.00 & -5.00 & 60.00 \\
\hline Admission FIM-M & 39.16 & 11.13 & 39.00 & 19.00 & 65.00 \\
\hline Discharge FIM-C & 25.89 & 6.13 & 27.00 & 13.00 & 35.00 \\
\hline$\Delta$ FIM-C & 3.91 & 3.90 & 3.00 & -1.00 & 17.00 \\
\hline FIM-E & 1.30 & 0.84 & 1.28 & -0.28 & 3.67 \\
\hline
\end{tabular}

Table 2. Functional Outcomes as Stratified by Ancillary Treatment Received during Inpatient Rehabilitation Program

\begin{tabular}{|c|c|c|c|c|}
\hline Statistically Significant Results $(\mathrm{p}<0.05)$ & Chemotherapy and Radiation & Radiation Alone & Chemotherapy Alone & No Treatment \\
\hline Total Patients (\%) & $9(20 \%)$ & $13(29 \%)$ & $3(7 \%)$ & $20(44 \%)$ \\
\hline FIM-D & 5.02 & 3.88 & 4.44 & 4.82 \\
\hline$\Delta$ FIM-T & 27.44 & 10.15 & 27.00 & 20.70 \\
\hline$\Delta$ FIM-M & 22.33 & 8.00 & 22.67 & 16.25 \\
\hline Discharge FIM-M & 63.22 & 42.77 & 56.00 & 58.35 \\
\hline FIM-E & 1.84 & 0.81 & 1.62 & 1.35 \\
\hline
\end{tabular}


Table 3. Functional Outcomes as Stratified by Discharge Disposition

\begin{tabular}{|c|c|c|c|}
\hline $\begin{array}{c}\text { Statistically Significant } \\
\text { Results (p< 0.05) }\end{array}$ & $\begin{array}{c}\text { Continued Aggressive } \\
\text { Treatment at Home }\end{array}$ & $\begin{array}{c}\text { Hospice House or Home } \\
\text { with Hospice Care }\end{array}$ & $\begin{array}{c}\text { Palliative Care at Skilled } \\
\text { Nursing Facility }\end{array}$ \\
\hline \hline Total Patients (\%) & $33(75 \%)$ & $8(18 \%)$ & $3(7 \%)$ \\
\hline$\Delta$ FIM & 1.22 & 0.29 & 0.51 \\
\hline FIM-D & 4.85 & 3.68 & 6.09 \\
\hline$\Delta$ FIM-T & 23.85 & 6.25 & 67.00 \\
\hline Discharge FIM-T & 86.45 & 63.13 & 5.00 \\
\hline$\Delta$ FIM-M & 19.33 & 3.25 & 44.00 \\
\hline Discharge FIM-M & 59.33 & 39.75 & 0.25 \\
\hline FIM-E & 1.54 & 0.47 & \\
\hline
\end{tabular}

Patients who received chemotherapy and radiation during inpatient rehabilitation had the greatest statistically significant improvement in $\triangle \mathrm{FIM}$, FIM-D, $\triangle \mathrm{FIM}-\mathrm{T}$, discharge FIM-T, discharge FIM-M, and FIM-E. Though $\Delta$ FIM-M was comparable to chemotherapy alone groups, discharge FIM-M was substantially greater for the chemotherapy and radiation group (Table 2). For those individuals who opted to continue aggressive oncological treatment at home after discharge, scores for $\triangle \mathrm{FIM}, \mathrm{FIM}-\mathrm{D}$, $\Delta$ FIM-T, discharge FIM-T, $\Delta$ FIM-M, discharge FIM-M, and FIM-E were substantially greater compared to individuals who either discharged to hospice or SNF (Table 3). Statistically significant correlations were noted between several variables evaluated in this review (Table 5).

\section{DISCUSSION}

As therapeutic interventions have evolved, there has been a growing interest in understanding the overall efficacy of individual brain tumor rehabilitation programs, given the very specific medical considerations for this patient population. Inpatient rehabilitation has shown benefits

Table 4. Ancillary Treatment while at Rehabilitation Compared to Discharge Disposition

\begin{tabular}{|c|c|c|c|}
\hline $\mathbf{p}<\mathbf{0 . 0 0 9}$ & Hospice Care & Palliative Care at SNF & Continued Aggressive Treatment at Home \\
\hline \hline No Treatment & $2(10 \%)$ & $1(5.0 \%)$ & $17(85 \%)$ \\
\hline Chemotherapy & 0 & 0 & $3(100 \%)$ \\
\hline Radiation & $6(50 \%)$ & $2(16.7 \%)$ & $4(33.3 \%)$ \\
\hline Chemo and Radiation & 0 & 0 & $9(100 \%)$ \\
\hline
\end{tabular}

Table 5. Statistically Significant Correlation Data

\begin{tabular}{|c|c|c|c|c|c|}
\hline $\mathrm{p}<0.05^{*}$ & LOS & Age & Change in Function Measures & Admission Data & Discharge Data \\
\hline $\begin{array}{c}\text { Correlation } \\
\text { Values }\end{array}$ & $\begin{array}{c}-0.463 \\
\text { (Admission FIM-T) } \\
\\
-0.536 \\
\text { (Admission FIM-M) } \\
\\
0.306 \\
(\Delta \mathrm{FIM}) \\
\\
-0.434 \\
(\mathrm{FIM}-\mathrm{E})\end{array}$ & $\begin{array}{c}-0.295 \\
\text { (FIM-D) } \\
\\
\\
-0.340 \\
\text { (Discharge FIM-T) }\end{array}$ & $\begin{array}{c}0.482 \\
\text { (Admission FIM-T and } \Delta \text { FIM-T) } \\
0.296 \\
\text { (FIM-A and } \Delta \text { FIM }) \\
0.942 \\
(\Delta \text { FIM-M and } \Delta \text { FIM-T) } \\
0.535 \\
(\Delta \text { FIM-C and } \Delta \text { FIM-T) } \\
0.679 \\
(\Delta \text { FIM-M and FIM-E) } \\
0.678 \\
(\Delta \text { FIM-T and FIM-E })\end{array}$ & $\begin{array}{c}0.843 \\
\text { (Admission FIM-T and } \\
\text { Admission FIM-M) } \\
0.646 \\
\text { (Admission FIM-T and } \\
\text { Admission FIM-C) }\end{array}$ & $\begin{array}{c}0.924 \\
\text { (Discharge FIM-T and } \\
\text { Discharge FIM-M) } \\
0.666 \\
\text { (Discharge FIM-C and } \\
\text { Discharge FIM-T) } \\
0.679 \\
\text { (Discharge FIM-M and } \\
\text { FIM-E) } \\
0.621 \\
\text { (Discharge FIM-T and } \\
\text { FIM-E) }\end{array}$ \\
\hline
\end{tabular}


relative to FIM score and functional gains for the cancer population in general without negative functional effects from concurrent radiation therapy or metastatic disease [21]. Interestingly, for those patients receiving radiation therapy for metastatic disease or primary brain tumors, greater functional gains were made compared to those patients not receiving radiation therapy [20]. However these results are not consistent, as it has also been shown that functional outcome with radiation were worse compared to individuals not receiving radiation [13]. The results of our study, however, show the least functional gain and the least improved functional score at discharge for radiation alone patients. A possible explanation was that patients who received radiation alone did so for palliative treatment and symptomatic relief, without intention for cure. Another consideration is that the radiation itself was not causing detrimental effects. Rather multimodal treatments for aggressive disease, such as narcotics for pain relief, benzodiazepines for anxiety, or anticholinergic medications for mood, could have impeded functional return due to side effects from these interventions [13]. Hence the individuals' medical and functional statuses prior to rehabilitation may have been poorer, with diminished expectations for significant functional and medical improvement. Patients who participated in hospice or SNF programs after discharge had less impressive functional gains as well. These dispositions may have reflected worsening medical or functional status due to a lack of response to oncological management or tumor progression in spite of intervention, which in turn negatively affect functionality.

Motor FIM gains were significant after acute inpatient rehabilitation for glioblastoma multiforme, brain metastases, and other brain tumors [22]. Patients with brain metastases had higher admission total FIM and motor FIM scores, whereas patients with glioblastoma multiforme had higher cognitive FIM scores overall. Though this study confirms that all patients with a brain tumor diagnosis can be expected to make functional gains, the greatest improvement was found in patients receiving concurrent chemotherapy and radiation therapy during the inpatient rehabilitation program, as well as in individuals who returned home to continue aggressive oncological management as outpatients. These individuals on average achieved supervision functional levels at the completion of their rehabilitation program. For these groups, discharge FIM-T scores were comparable to previously reported results, though FIM-E was generally better $[14,16,19,21,23]$. This may indicate that aggressive interventions with both medical and radiation oncology could lead to improved functionality from decreased mass effects from the tumor. In addition, radiation therapy has shown increased potentiation effects with concurrent administration of oral temozolomide, which in turn could have led to further tumor burden reduction. The results of this study may indicate that individuals with higher grade tumors can still be expected to make significant functional improvements with concurrent aggressive oncological treatment, which then can facilitate transition to home and decreased physical burden of care for families. For those patients who did not receive chemotherapy or radiation therapy, similar functional gains may be expected as compared to treatment groups. This may seem counterintuitive given that tumor burden persists, however, the functional gains may have been balanced by decreased medical side effects from acute oncological ancillary treatment.

Though it has been found that high dose dexamethasone administration, organ metastases, and increased brain lesions may indicate poorer prognosis, rehabilitation interventions have still shown improved functional gains [22]. However, concern of the establishment of brain tumor specific inpatient rehabilitation programs has been related to medical complexity, prognosis, and increased rate of transfer to acute care for medical treatment; only $11.3 \%$ of our patients required acute medical transfer, which was significantly better than previously reported results [24]. This may be reflective of the collaboration between physicians from both acute care and rehabilitation to prevent the exacerbation of medical co-morbidities through aggressive monitoring and medical management while in rehabilitation and while receiving concurrent oncological treatment. Our inpatient rehabilitation facility has dedicated oncology rehabilitation services, and co-management with medical, radiation, and surgical oncology services are common during the inpatient rehabilitation stay. All patients in this study were cared for by physiatrists, nurses, and therapists with established specialization in oncology rehabilitation. With this collaboration, acute oncological care is coordinated such that patients can discharge to home with plans of aggressive outpatient oncological follow-up to maximize their QOL, functionality, and survival.

When comparing the functional outcomes of low grade versus high grade astrocytomas, patients with higher grade astrocytoma generally had greater LOS and greater relative gain in FIM scoring, possibly indicating that longer LOS could translate into improved function [23]. Our length of stay was 18.13 , which was decreased compared to previously reported results $[14,19,20,22,23]$. This may be a function of neurological recovery after space occupying lesions are surgically excised [19]. In this study, increased LOS correlated with increased $\Delta$ FIM. However individuals with increased LOS may have had functional and medical deficits that were more serious based on the severity of their disease; this may have accounted for decreased admission scores for FIM-M and FIM-T, as well as negative correlation with FIM-E.

Though long term steroid use can have detrimental effects, short term aggressive steroid treatment can have positive effects related to improved cerebral edema and neurological recovery which in turn may have led to higher FIM scoring in this study. Relatively diminished improvement in FIM score for brain tumor patients compared to other patient populations presented in previous studies may have been secondary to ancillary treatments (including chemotherapy, radiation therapy, and surgery), which all have known side effects, such as fatigue, malaise, nausea, vomiting, and generalized debility, that can affect function. Improved medications and treatment options for these side effects may have allowed patients in this study who received chemotherapy and radiation therapy to achieve improved functional outcomes at the completion of their rehabilitation stay. The average age of patients evaluated in these previous studies ranged from 53.1 to 59.9 years old, which is comparably younger than our patient sample. For 
this review, the median age was 70 , with an average age of 65 . This study confirmed improved functional outcomes can be expected in a patient population that was overall older than previously described, though correlation data confirms that older age yields less positive results.

Our results confirmed that FIM-M scores overall correlated strongly with FIM-T scores overall, possibly indicating that FIM-M may be a strong indicator when anticipating outcomes in terms of FIM-T. FIM-C data also correlates with FIM-T data but with a weaker relationship than FIM-M. Future work is necessary to understand how antidepressant and neurostimulant use may affect FIM-C. In addition, results from FIM-E correlation data were expected. As discharge FIM-M and FIM-T scores improve, the efficiency of rehabilitation gains per day also improves, but as LOS increases, the daily FIM improvement decreases. Admission FIM-T and FIM-A may have been positively associated with $\triangle$ FIM-T and $\triangle$ FIM respectively due to selection bias on admission of generally higher functioning patients who would be expected to have better functional outcomes.

There are several limitations to this study. Selection bias of patients with generally higher functional levels may have led to better overall outcomes and tolerance to the negative side effects of ancillary treatment. Specific attention to discharge disposition and family support may have also improved our rate of discharge to home. In addition, our patient population benefits from treatment at a major regional cancer center with ample support services from medical, radiation, and surgical oncology. This allowed for admission of patients with significant medical complexity from neurological deficits associated with brain tumors as well as side effects from ancillary treatment. Due to the retrospective nature of this study, causation could not be established with our correlation data, specifically as it relates to the impact of FIM outcomes to acute oncological plan of care and discharge disposition. Prospective trials may be useful to understand how FIM scores may directly influence both oncological and discharge planning. Since there was not a longitudinal collection of data reflecting functional status after discharge from acute inpatient rehabilitation, conclusions could not be made regarding the sustainability of functional gains achieved while in rehabilitation. Furthermore, our relatively low sample size limits the generalizability of these results. Prospective trials with larger sampling sizes could help stratify functional outcomes by the following: brain tumor diagnosis; individual therapy disciplines; tumor location; complications from resection; duration and specificity of ancillary treatment; socioeconomic status; patient support after discharge; oncological support services while in rehabilitation; and availability of regional oncological services in general for treatment. Finally, consideration should be made for the use of alternative measurement tools related to cognition, function, mood, and quality of life as these may provide additional information that could help guide comprehensive treatment plans and improve outcomes.

\section{CONCLUSION}

The results of this study indicate positive findings regarding the functional outcomes for patients after acute inpatient rehabilitation programs specifically designed for the brain tumor population, for all discharge dispositions and ancillary treatment plans considered in this study. Patients who received both chemotherapy and radiation therapy during acute inpatient rehabilitation had the greatest functional improvement at the time of discharge as compared to other ancillary treatment groups. Patients who discharged to home to pursue further aggressive treatment had the greatest functional improvement compared to other discharge dispositions. These finding are important in understanding factors that can facilitate discharge to home, and thus allow for further aggressive oncological treatment with potentially positive implications regarding function, QOL, and survival.

\section{CONFLICT OF INTEREST}

The authors declare that they have no competing interests.

\section{ACKNOWLEDGEMENTS}

\author{
Declared none.
}

\section{REFERENCES}

[1] Maity A, Pruitt AA, Judy KD, Phillips PC, Lustig R. Cancer of the central nervous system. In: Abeloff MD, Armitage JO, Niederhuber JE, Kastan MB, McKenna WG, Eds. Abeloff's clinical oncology, $4^{\text {th }}$ ed. Philadelphia: Churchill Livingstone 2008. pp. 1075-127.

[2] Wen P, Kesari S. Malignant gliomas in adults. N Engl J Med 2008; 359(5): 492-507.

[3] Laws ER, Parney IF, Huang W, et al. Glioma Outcomes Investigators. Survival following surgery and prognostic factors for recently diagnosed malignant glioma: data from the glioma outcomes project. J Neurosurg 2003; 99(3): 240-4.

[4] DeAngelis L. Brain tumors. N Engl J Med 2008; 344(2): 114-23.

[5] Barnholtz-Sloan JS, Sloan AE, Schwartz AG. Cancer of the brain and other central nervous system. In: Ries LAG, Young JL, Keel GE, Eisner MP, Lin YD, Horner M-J, Eds. SEER survival monograph: cancer survival among adults: U.S. SEER program, 1988-2001, patient and tumor characteristics. Bethesda: National Cancer Institute 2007; pp. 203-16.

[6] Bartolo M, Zucchella C, Pace A, et al. Early rehabilitation after surgery improves functional outcome in inpatients with brain tumours. J Neurooncol 2012; 107(3): 537-44.

[7] Pirracchio R, Resche-Rigon M, Bresson D, et al. One-year outcome after neurosurgery for intracranial tumor in elderly patients. J Neurosurg Anesthesiol 2010; 22(4): 342-6.

[8] Formica V, Del Monte G, Giacchetti I, et al. Rehabilitation in neuro-oncology a meta-analysis of published data and a monoinstitutional experience. Integr Cancer Ther 2011; 10(2): 119-26.

[9] Gabanelli P. A rehabilitative approach to the patient with brain cancer. Neurol Sci 2005; 26(Suppl 1): S51-2.

[10] Hill CI, Nixon CS, Ruehmeier JL, Wolf LM. Brain tumors. Phys Ther 2002; 82(5): 496-502.

[11] Giordana MT, Clara E. Functional rehabilitation and brain tumour patients. a review of outcome. Neurol Sci 2006; 27(4): 240-4.

[12] Mukand JA, Blackinton DD, Crincoli MG, Lee JJ, Santos BB Incidence of neurologic deficits and rehabilitation of patients with brain tumors. Am J Phys Med Rehabil 2001; 80(5): 346-50.

[13] O’Dell MW, Barr K, Spanier D, Warnick RE. Functional outcomes of inpatient rehabilitation in persons with brain tumors. Arch Phys Med Rehabil 1998; 79(12): 1530-4.

[14] Huang ME, Cifu DX, Keyser-Marcus L. Functional outcomes in patients with brain tumor after inpatient rehabilitation: comparison with traumatic brain injury. Am J Phys Med Rehabil 2000; 79(4): 327-35.

[15] Geler-Kulcu D, Gulsen G, Buyukbaba E, Ozkan D. Functional recovery of patients with brain tumor or acute stroke after rehabilitation: a comparative study. J Clin Neurosci 2009; 16(1): 74-8. 
[16] Greenberg E, Treger I, Ring H. Rehabilitation outcomes in patients with brain tumors and acute stroke. Am J Phys Med Rehabil 2006; 85(7): 568-73.

[17] Karakaya M, Kose N, Otman S, Ozgen T. Investigation and comparison of the effects of rehabilitation on balance and coordination problems in patients with posterior fossa and cerebellopontine angle tumours. J Neurosurg Sci 2000; 44(4): 2205 .

[18] Wesling M, Brady S, Jensen M, Nickell M, Stratkus D, Escobar N. Dysphagia outcomes in patients with brain tumors undergoing inpatient rehabilitation. Dysphagia 2003; 18(3): 203-10.

[19] Huang ME, Cifu DX, Keyser-Marcus L. Functional outcome after brain tumor and acute stroke: a comparative analysis. Arch Phys Med Rehabil 1998; 79(11): 1386-90.
[20] Marciniak CM, Sliwa JA, Heinemann AW, Semik PE. Functional outcomes of persons with brain tumors after inpatient rehabilitation. Arch Phys Med Rehabil 2001; 82(4): 457-63.

[21] Marciniak CM, Sliwa JA, Spill G, Heinemann AW, Semik PE. Functional outcome following rehabilitation of the cancer patients. Arch Phys Med Rehabil 1996; 77(1): 54-7.

[22] Tang V, Rathbone M, Park DJ, Jiang S, Harvey D. Rehabilitation in primary and metastatic brain tumours, impact of functional outcomes on survival. J Neurol 2008; 255(6): 820-7.

[23] Fu JB, Parsons HA, Shin KY, et al. Comparison of functional outcomes in low- and high-grade astrocytoma rehabilitation inpatients. Am J Phys Med Rehabil 2010; 89(3): 205-12.

[24] Alam E, Wilson RD, Vargo MM. Inpatient cancer rehabilitation: a retrospective comparison of transfer back to acute care between patients with neoplasm and other rehabilitation patients. Arch Phys Med Rehabil 2008; 89(7): 1284-9.

Received: July 23, 2012

Revised: September 25, 2012

Accepted: September 26, 2012

(C) Raj et al.; Licensee Bentham Open.

This is an open access article licensed under the terms of the Creative Commons Attribution Non-Commercial License (http://creativecommons.org/licenses/by-nc/3.0/) which permits unrestricted, non-commercial use, distribution and reproduction in any medium, provided the work is properly cited. 